\title{
RESET
}

Recherches en sciences sociales sur Internet

\section{Les nouvelles thérapies par Internet}

New Internet-based Therapies

\section{Jan Bergström}

\section{(2) OpenEdition}

\section{Journals}

Édition électronique

URL : http://journals.openedition.org/reset/124

DOI : 10.4000/reset.124

ISSN : 2264-6221

\section{Éditeur}

Association Recherches en sciences sociales sur Internet

\section{Référence électronique}

Jan Bergström, «Les nouvelles thérapies par Internet », RESET [En ligne], 2 | 2013, mis en ligne le 01 juillet 2013, consulté le 09 octobre 2020. URL : http://journals.openedition.org/reset/124 ; DOI : https://doi.org/10.4000/reset.124

Ce document a été généré automatiquement le 9 octobre 2020.

(c) Association Recherches en sciences sociales sur Internet 


\title{
Les nouvelles thérapies par Internet
}

\author{
New Internet-based Therapies
}

Jan Bergström

\section{Introduction}

1 Depuis les débuts d'Internet, les sujets relatifs à la santé mentale ont fait l'objet de nombreuses appropriations de la part des internautes qui, par e-mails ou par chats, sur des forums ou des sites web, ont témoigné de leurs souffrances psychiques, ont soulevé des questions, ont cherché des informations et du soutien. Il n'est pas surprenant que les individus touchés par des troubles psychiques aient devancé les professionnels et les institutions sanitaires dans l'usage de cette nouvelle technologie. Néanmoins, depuis une quinzaine d'années, ces derniers sont entrés en jeu: les organismes publics de santé, puis dans un second temps la psychiatrie, ont investi Internet, proposant des informations concernant les troubles psychiques, des formulaires de dépistage et des conseils. Internet a d'abord été considéré par ces institutions comme un simple "auxiliaire ", le cœur du système de la santé mentale continuant d'être pratiqué de façon traditionnelle par le biais de thérapies en face à face. Or, depuis environ dix ans, Internet est également envisagé comme un espace où peuvent se pratiquer la psychothérapie et le traitement psychologique ${ }^{1}$.

2 Il existe aujourd'hui de nombreux dispositifs utilisant Internet comme un moyen d'intervention auprès de personnes souffrants de troubles mentaux, tels que la dépression ou les troubles anxieux. D'une part, il s'agit de démarches visant à faire d'Internet un média alternatif pour le thérapeute souhaitant effectuer une thérapie traditionnelle à distance. Les éléments constitutifs de la thérapie restent ici inchangés. C'est le cas de la thérapie par e-mails, souvent appelée e-therapy (Manhal-Baugus, 2001), de la thérapie par chat (Rassau \& Arco, 2003) et de la thérapie, ou de l'intervention psychiatrique, par vidéo («télépsychiatrie»; Ruskin et al., 2004). Certains psychothérapeutes voient dans Internet la possibilité d'offrir, par exemple, une thérapie psychanalytique à distance par l'un de ces moyens. Si une nouvelle technologie est introduite dans ces soins, si le moyen de la transmission est différent, 
ce qui est compris comme le fondement du changement thérapeutique - la relation psychothérapeutique entre patient et thérapeute - reste toutefois le même.

D'autre part, il existe de nouvelles formes thérapeutiques dans lesquelles Internet change plus profondément les éléments constitutifs de la thérapie : celles qui engagent la substitution partielle ou totale d'un programme informatique de self-help au thérapeute (Baer, Greist \& Marks 2007; Andersson, 2009). Dans ces cas, l'usage d'Internet ne traduit pas seulement une autre façon de transmettre la psychothérapie. Il modifie la nature même de cette dernière. La thérapie, ou plus précisément le changement thérapeutique, n'est plus assurée par un thérapeute mais par un programme de self-help, et le rôle de thérapeute n'est que de faciliter le suivi du programme.

4 L'objectif principal de cette deuxième démarche consiste à augmenter l'accessibilité de la psychothérapie, c'est-à-dire réduire les obstacles que peuvent rencontrer les patients lorsqu'ils souhaitent faire appel à un dispositif de soin. Cette question constitue un défi majeur pour de très nombreux systèmes de soins nationaux (Richards, Lovell \& McEvoy, 2003). La seule manière pour une personne d'avoir accès à une thérapie traditionnelle est souvent de recourir à un thérapeute exerçant dans le secteur privé. Cela n'est cependant possible que pour des individus disposant de ressources économiques relativement importantes. Or, l'accessibilité s'améliore avec les programmes de self-help, dans la mesure où ces derniers rendent la thérapie moins dépendante de la disponibilité des thérapeutes: en diminuant le temps que consacre chaque thérapeute à chaque patient, un plus grand nombre de patients peuvent accéder à un traitement (Andersson, 2009).

De ce point de vue, le premier type de thérapies à distance (la thérapie par e-mail, chat ou vidéo) n'accroît pas l'accessibilité des soins. Il appartient toujours au thérapeute de transmettre la thérapie, ce qui nécessite autant de temps qu'en en face à face. Cette démarche n'augmente donc pas les "ressources en psychothérapie » dans un système de soins, en ne permettant pas à un plus grand nombre de personnes d'y avoir accès. Ces thérapies, qui restent classiques bien que transmises avec l'aide d'un média alternatif, semblent également problématiques d'un point de vue scientifique dans la mesure où il n'existe que très peu, voire pas du tout, d'études cliniques ayant évalué leur efficacité.

Ces faiblesses sont les qualités des seconds types de démarche, habituellement intitulées Internet-based guided self-help ou, plus précisément, Internet-based psychological treatment (Traitement psychologique par Internet). Pour ces raisons, l'usage thérapeutique d'Internet ayant reçu le plus d'attention ces dernières années est celui du traitement psychologique par Internet. Ce dernier devient en effet, au cours de la dernière décennie, un champ de recherche en pleine expansion en psychologie clinique, en particulier aux Etats-Unis, en Grande-Bretagne, aux Pays-Bas, en Australie et en Suède. Plusieurs études montrent l'efficacité de ces traitements en ligne dans l'assistance aux personnes souffrant notamment des troubles psychiques les plus répandus, à savoir la dépression et les troubles anxieux (Andersson \& Cuijpers, 2009; Cuijpers et al., 2009). De façon plus surprenante, des études cliniques indiquent que ce type de soin serait aussi efficace pour ces troubles que des thérapies dispensées de manière traditionnelle (Bergström et al., 2010 ; Cuijpers et al., 2010). La question de la durée de cette efficacité étant importante, dans le cas des soins par Internet, des 
patients ont été suivis jusqu'à cinq ans après la fin de leur prise en charge, et l'on a ainsi pu observer la durabilité des effets thérapeutiques (Hedman et al., 2011b).

Cet article propose une synthèse du développement scientifique du traitement psychologique par Internet, en explicitant et discutant ses traits caractéristiques. Nous y comparerons ce type de soin avec la psychothérapie traditionnelle et dessinerons les enjeux que revêtent les soins sur Internet dans le champ de la psychothérapie; avant tout au niveau théorique mais aussi au niveau pratique. Nous mettrons plus précisément en évidence les nombreux facteurs indiquant que les thérapies basées sur Internet constituent, cent ans après la naissance de la discipline, un défi profond et durable à la psychothérapie.

8 A noter, nous désignerons par «psychothérapie traditionnelle » toute psychothérapie pratiquée en face à face, quelle que soit sa base théorique (psychanalytique, cognitive/ comportementale ou autre). Cependant, nous verrons qu'il existe une relation très claire entre le développement des nouveaux traitements en ligne d'une part, et le courant cognitif et comportemental d'autre part. Le fait que les nouvelles thérapies par Internet soient développées dans des pays où le courant cognitif et comportemental constitue désormais le paradigme dominant n'est donc pas un hasard. Ce n'est pas non plus un hasard que l'auteur de cet article, ayant conduit des recherches dans ce domaine, soit attaché à la psychothérapie cognitive et comportementale, orientation qui inévitablement se reflète dans ce texte.

\section{L'histoire du self-help dans le domaine du traitement psychologique}

Bien avant l'apparition des traitements psychologiques par Internet, des chercheurs extérieurs au domaine de la psychothérapie traditionnelle se sont intéressés à l'utilisation de différents moyens écrits et techniques pour intervenir auprès de personnes souffrants de troubles mentaux. Dans les années 1960, Joseph Weizenbaum (1966) a ainsi développé le programme informatique «ELIZA », qui était censé générer les réponses d'un psychothérapeute «non-directif» (dans la tradition de Carl Rogers). Le patient se retrouvait donc « en session » avec un ordinateur. Si ce fameux exemple avait en réalité, au moins partiellement, un objectif parodique, il esquissait déjà quelques pistes pour un champ de recherche à venir: l'utilisation d'ordinateurs permettant de remplacer le psychothérapeute.

\section{Les débuts des thérapies informatisées}

Suite à l'attention croissante des chercheurs en psychiatrie pour les ordinateurs dans les années 1970 (Klein, Greist \& Van Cura, 1975), certains se sont intéressés au cours des années 1980 à l'usage des technologies informatiques dans le dépistage des troubles et le recueil d'informations auprès des patients (Carr \& Ghosh, 1983). L'étape suivante l'utilisation des ordinateurs dans un but thérapeutique - n'était alors pas loin, en particulier pour les chercheurs en psychiatrie dont le cadre théorique était plutôt comportemental. Parmi eux, on peut citer Isaac Marks, futur professeur de psychiatrie reconnu à l'Institute of Psychiatry de Londres, qui a conçu avec ses collaborateurs un programme d'«auto-exposition » pour les troubles anxieux (Ghosh, Marks \& Carr, 1984). L'« exposition", mécanisme central dans les thérapies comportementales, 
désigne le processus au cours duquel le patient se rapproche graduellement de ce qui lui fait peur afin de surmonter peu à peu sa crainte. Cette étude de Marks et ses collègues constitue le premier exemple publié de ce qui sera ensuite appelé la computerised cognitive behaviour therapy (CCBT), littéralement «thérapie comportementale et cognitive informatisée » (Baer, Greist \& Marks 2007). Tout comme pour le célèbre ELIZA vingt ans plus tôt, l'idée était de remplacer le thérapeute par un programme informatique : "l'ordinateur était programmé pour 'discuter' d'exercices d'exposition » (Ghosh, Marks \& Carr, 1984). Autrement dit, il s'agissait d'un programme de pur self-help, sans aucun contact thérapeutique.

11 Les travaux de Marks et des chercheurs ayant suivi ses pas ont donné naissance à plusieurs programmes interactifs en anglais, dont notamment Fear Fighter pour les troubles d'anxiété et Beating the Blues pour la dépression. Ces deux programmes ont montré leur efficacité dans la réduction des symptômes d'anxiété et de dépression (Kenwright, Liness \& Marks, 2001; Proudfoot et al., 2004), bien que de nombreux patients semblent ne pas aller jusqu'au bout de ces dispositifs non-guidés. Comme leurs noms « accrocheurs » le suggèrent, ces programmes n'ont pas été développés dans un dessein uniquement scientifique, mais bel et bien dans un but commercial. Ils ont depuis leur création été intégrés à certains systèmes de soin, en Grande-Bretagne, par exemple, où les entreprises informatiques qui les ont développés ont signé des contrats publics (Palmili \& Marks, 2009).

\section{La « bibliothérapie »}

12 Une autre tradition a également eu une grande importance dans l'histoire du traitement psychologique par Internet. Il s'agit de la tradition souvent appelée "bibliothérapie». Ce terme désignait initialement tous les moyens écrits, textes littéraires inclus, utilisés afin de diminuer une souffrance physique ou psychologique (Alston, 1962). A partir des années 1970, le terme se voit plus clairement utilisé pour désigner des livres "d'auto-assistance", écrits comme des manuels de self-help pour différents troubles psychiques, avant tout pour la dépression et les troubles d'anxiété. Ces démarches ont-elles aussi été construites dans une orientation comportementaliste. Sont ainsi publiées de plus en plus d'études montrant l'efficacité de ces textes de self-help, notamment pour diminuer les symptômes d'anxiété et de dépression (Goldiamond, 1976; Rosen, Glasgow \& Barrera, 1976). Dans ce courant de recherche, il n'était pas question de complètement supplanter le thérapeute mais de modifier son rôle. Celui-ci n'était pas envisagé comme le fondement de la thérapie, mais comme quelqu'un qui pourrait assister le patient dans le suivi d'un manuel de selfhelp. Autrement dit, le thérapeute restait présent, mais moins que lors d'une thérapie traditionnelle et de façon différente; les brefs contacts avec les patients pouvaient aussi bien, par exemple, se dérouler par téléphone.

\section{Self-help et processus thérapeutique}

13 L'utilisation du self-help s'inscrit donc à ses prémisses dans un continuum allant du pur self-help - les self-administered treatments - aux traitements où le thérapeute transmet tout de même la thérapie - les therapist-administered treatments (Glasgow \& Rosen, 1978). Si l'on regarde ces termes de plus près, on s'aperçoit que dans les deux cas, les traitements sont qualifiés d'administrés (transmis) : on décrit un contenu, une matière 
thérapeutique déjà existante, qui pourrait être "administré » au patient avec ou sans thérapeute. Cela contraste avec la conceptualisation traditionnelle du processus thérapeutique dans lequel la thérapie sans thérapeute est inconcevable. Que désigne cette «matière » thérapeutique qui existe indépendamment d'un thérapeute et qui est décrite comme "extérieure» à la relation psychothérapeutique? Il s'agit tout simplement d'un manuel : un texte décrivant les démarches à suivre afin, par exemple, de s'exposer à des stimuli dont on a peur ou se remettre graduellement d'un état dépressif. Une autre différenciation cruciale qu'il importe de faire ici distingue la thérapie avec manuel et la thérapie sans manuel. Ou, dans les termes de Glasgow \& Rosen (1978), les therapist administered treatments (le thérapeute suit un manuel) et les therapist directed treatments (aucun manuel n'est utilisé et la totalité de la thérapie est fondée sur le contact/processus thérapeutique individuel).

\section{Développements précurseurs du traitement psychologique par Internet}

Dans les années 2000, deux processus parallèles conduisent au développement de ce que l'on appelle aujourd'hui le traitement psychologique par Internet. Le premier est le changement des usages sociaux des ordinateurs. Si les utilisateurs de ces derniers étaient auparavant mis en contact avec des programmes informatiques, ils se trouvent de nos jours en contact avec d'autres personnes, par e-mails, réseaux sociaux, chats, etc.

L'autre processus est d'ordre scientifique. L'usage "non-guidé » (sans le soutien d'un thérapeute) des programmes de self-help a de plus en plus été mis en cause. Plusieurs études ont en effet montré qu'une grande partie des utilisateurs des programmes mentionnés ci-dessus ne les suivaient pas jusqu'au bout. Il n'a par ailleurs pas été établi que les effets de ces programmes dépassent l'effet de la simple lecture d'informations par le patient (Christensen, Griffiths \& Jorm, 2004 ; Christensen et al, 2006 ; Andersson \& Cuijpers, 2009).

Ces deux processus (sociétal et scientifique) ont conduit au développement des traitements contemporains sur Internet, qui tirent profit des avantages de ce média par la possibilité d'un bref contact thérapeutique en ligne pour compléter le contenu principal du soin (le texte du self-help). Il a été montré que ce contact thérapeutique, même court, augmente les chances que le patient finisse le programme et qu'il améliore nettement l'efficacité du suivi psychologique (Palmqvist, Carlbring \& Andersson, 2007 ; Titov et al., 2008). C'est la naissance de ce qui sera qualifié d'Internet-based guided selfhelp ou d'Internet-based psychological treatment (Traitement psychologique par Internet).

\section{Le traitement psychologique par Internet}

Plus concrètement, en quoi consiste le traitement psychologique par Internet décrit jusqu'ici ? Nous allons désormais caractériser ce type de thérapie, en commençant par présenter ses fondements théoriques et ses dispositifs concrets ${ }^{2}$. Cette caractérisation va inévitablement nous mener à discuter des défis que cette évolution pose aux psychothérapies traditionnelles. 


\section{Fondements théoriques} Dans le domaine des troubles anxieux, l'exposition constitue la démarche thérapeutique principale, si l'on suit une orientation comportementale (Barlow, 2002). Cette orientation se fonde théoriquement sur la caractérisation des troubles comme des problèmes de conditionnement dysfonctionnel. Plus précisément, " conditionnement » signifie que l'origine de la peur ou de l'anxiété se trouve dans une histoire personnelle d'“apprentissage " anxieux. Ces émotions ont été conditionnées à (c'est-à-dire associées à) des stimuli spécifiques, tels que les transports en commun ou les lieus clos dans le cas de l'agoraphobie, les situations sociales d'interaction avec d'autres individus dans le cas de l'anxiété sociale ou la "saleté » dans le cas de certains troubles obsessionnels compulsifs (Barlow, 2002). Puisque la peur se fonde sur un " apprentissage ", les démarches thérapeutiques proposent un "réapprentissage ", où la personne s'expose graduellement aux stimuli anxiogènes afin de s'y habituer (ce qui produit une baisse graduelle d'anxiété). Lors d'une thérapie comportementale traditionnelle, ces démarches sont décrites et introduites par le thérapeute, et souvent effectuées par le patient et le thérapeute ensemble, dans le bureau du thérapeute ou à l'extérieur (Barlow, 2002). Lorsque le traitement se produit par Internet, les mêmes démarches sont décrites par un texte et menées à bien par le patient dans sa vie quotidienne, avec l'aide des formulaires et d'autres moyens interactifs inclus dans le programme informatique; mais aussi, avec les brefs encouragements par e-mail du thérapeute, qui aide le patient à trouver des solutions aux problèmes pouvant survenir lors du traitement (Andersson et al., 2008)

En ce qui concerne les troubles dépressifs, les démarches thérapeutiques comportementales sont similaires dans le sens où elles se focalisent sur le fonctionnement concret et quotidien du patient. L'objectif est de rompre avec un mode de vie souvent passif, réactif et ruminatoire par des démarches d' « activation » visant à introduire dans la vie quotidienne du patient un mode de vie plus proactif. Cette méthode s'appelle la behavioral activation - l'«activation comportementale » (Martell, Addis \& Jacobson, 2001).

21 Un traitement psychologique est donc, de ce point de vue, conçu comme une expérience d'apprentissage pour le patient, où cours de laquelle ce dernier apprend à changer concrètement son mode de vie. Cette optique contraste avec la conception traditionnelle du processus psychothérapeutique, telle que mentionnée précédemment, qui considère que la relation psychothérapeutique est l'élément constitutif et nécessaire d'une psychothérapie réussie. En un sens, la possibilité de «formaliser » la thérapie en un manuel de self-help était depuis toujours présente dans la thérapie comportementale, même si pendant longtemps celle-ci se transmettait également d'une manière traditionnelle, par l'échange entre patient et thérapeute. En revanche, mais nous y reviendrons, l'idée d'abstraire les traits constitutifs d'une thérapie de la relation 
thérapeutique s'avère inconcevable selon les approches psychanalytiques et/ou psychodynamiques.

\section{Les programmes de self-help}

Précisons quelques caractéristiques des programmes de self-help, qui constituent le cœur du traitement psychologique par Internet. Ces programmes sont presque toujours spécifiques à un diagnostic en particulier. En effet, même si l'on considère que de grands principes, tels que la méthode d'exposition, sont généraux, les exercices et les démarches qui en découlent sont adaptés à chaque trouble. Il existe par conséquent des traitements consacrés au trouble de panique (Bergström et al., 2009; Wims et al., 2010), à la phobie sociale (Hedman et al., 2011a), au trouble obsessionnel compulsif (Andersson et al., 2012), à la dépression (Perini, Titov \& Andrews, 2009 ; Andersson et al., 2009). Les recherches les plus récentes ont débouché sur des programmes "transdiagnostiques ", qui fonctionnent à partir de principes pertinents dans le cas, par exemple, de toutes les différentes formes de troubles anxieux (Titov et al., 2010). Un autre développement récent concerne l'utilisation de smartphones (Ly et al., 2012), qui remplacent les ordinateurs en tant que moyen de présenter le programme de self-help, mais la démarche en soi reste la même.

Un programme de self-help se divise habituellement en "modules ", équivalents aux chapitres d'un livre (ce qui reflète son affinité avec la tradition bibliothérapeutique mentionnée précédemment). Le patient suit une progression graduelle à travers les modules successifs. Le premier module comporte des informations générales concernant la nature du trouble ou du diagnostic en question - on appelle cela la " psychoeducation ». Le suivant propose des exercices permettant au patient de déceler les situations problématiques de sa vie quotidienne. Viennent ensuite les modules de traitement à proprement parler, constitués d'exercices d'exposition ou d'activation. La partie principale d'un traitement consiste donc en des exercices que le patient doit réaliser au quotidien. Le programme est là pour aider l'individu, par des principes généraux, des fiches interactives, des exemples pédagogiques qui mettent en scène des patients fictifs suivant le programme. Tous ces outils visent à augmenter les chances que le patient parvienne à changer concrètement son mode de vie effectif et quotidien. Vers la fin du programme, le patient revoit le traitement parcouru, est encouragé à tirer des conclusions et travaille sur un plan de prévention d'éventuelles rechutes.

On reproche souvent aux soins par Internet de se dérouler uniquement devant l'ordinateur. En réalité, au regard de ces explications, le vrai changement thérapeutique se produit dans la vie quotidienne du patient. En cela, le traitement par Internet ne change rien au processus thérapeutique classique, du moins en comparaison aux thérapies comportementales ordinaires : même lorsque la thérapie est transmise en face à face, par le biais de séances hebdomadaires dans un cabinet, le changement thérapeutique se passe principalement en dehors $d u$ cabinet. Plus précisément, il s'opère lors de la vie quotidienne du patient, quand celui-ci s'entraîne à changer concrètement son comportement et sa manière d'aborder les situations problématiques.

Quelle est donc l'expérience des patients qui suivent un traitement psychologique par Internet? Des études qualitatives indiquent que cette expérience ressemble à celle de la thérapie transmise de façon traditionnelle. Cela pourrait indiquer que ce nouveau type 
de soin semble plus impersonnel à certains thérapeutes qu'aux patients eux-mêmes (Bendelin et al., 2011).

\section{La place du thérapeute}

Tout au long du traitement, le patient reste en contact avec un thérapeute qui le soutient, l'encourage et l'aide à trouver des solutions aux problèmes survenus pendant la réalisation des exercices. Si des moyens de communications divers existent pour accompagner le programme de self-help (téléphone, chat, etc.), les e-mails sont souvent privilégiés. Cette solution apparait plus pratique puisqu'il s'agit d'un outil de communication asynchrone qui ne nécessite pas de prise de rendez-vous (à la différence du téléphone ou du chat). Une fonctionnalité e-mail est par conséquent souvent intégrée dans le programme même, ce qui évite certains problèmes techniques mais aussi des problèmes de confidentialité liés à l'utilisation d'une adresse e-mail privée.

27 Les contacts par e-mails sont brefs. Souvent, ils ne durent pas plus de dix minutes par semaine au total, et se limitent à une aide donnée au patient pour qu'il puisse suivre le programme (Palmqvist, Carlbring \& Andersson, 2007 ; Bergström et al., 2010). Comme nous l'avons déjà souligné, l'essentiel du contenu thérapeutique se transmet par le biais du programme de self-help, et non par le thérapeute. La brièveté des contacts avec ce dernier répond alors à un objectif important, expliqué précédemment : pour pouvoir augmenter l'accessibilité d'un traitement, il faut qu'un même professionnel puisse s'occuper de plus de patients.

Notons enfin que parmi les études menées en vue d'évaluer l'efficacité de ce type de suivi (Cuijpers et al., 2010), les thérapeutes étaient presque exclusivement des étudiants en psychologie clinique, et non des psychologues ou des thérapeutes expérimentés ou en exercice. C'est un fait important, qui contraste à nouveau fortement avec les psychothérapies traditionnelles. Dans ces dernières, l'accent est mis sur la compétence du thérapeute et l'efficacité ou l'utilité de la thérapie est pensée comme étant largement dépendante de cette compétence thérapeutique. Dans un traitement par Internet, le moteur du processus thérapeutique est le programme de self-help, et non le thérapeute et ses compétences personnelles.

\section{Conclusion. Les défis posés à la psychothérapie traditionnelle}

Depuis l'émergence de la psychothérapie il y a environ cent ans, de très nombreuses thérapies ont été développées et pratiquées. Si ces thérapies ont pu avoir des fondements théoriques différents, deux éléments les réunissent en général : d'une part, des dispositions concrètes, comme les séances hebdomadaires, et d'autre part une conception du processus thérapeutique plaçant la relation thérapeute/patient comme dimension constitutive de la psychothérapie.

Si ces conceptions ont déterminé la définition de la psychothérapie dans son ensemble, c'est avant tout dans la théorie psychanalytique que le rôle du thérapeute et l'importance de la relation psychothérapeutique ont été théorisés comme des aspects essentiels du soin. Cette position repose sur la conception fondamentale que les effets 
curatifs de la thérapie dépendent de la relation thérapeutique (Muran \& Barber, 2010 ; Brusset, 2012). La psychothérapie est conçue comme un processus unique qui se produit dans la relation entre un patient et son thérapeute, au cours duquel se met en place une découverte psychique impossible sans cette relation. La psychologie du patient échappe à celui-ci dans sa vie quotidienne et ne peut être comprise que par une analyse, à partir de ce qui émerge dans la relation entre les deux protagonistes. La définition même de la psychothérapie repose alors sur un processus relationnel et subjectif (Muran \& Barber, 2010).

31 La conception comportementale du changement thérapeutique contraste fortement avec celle de la psychothérapie psychanalytique. Elle situe le cœur du changement thérapeutique non pas au sein de la relation avec le thérapeute mais dans la vie quotidienne du patient. Cette conception permet d'abstraire le processus thérapeutique de la séance ou de la relation entre patient et thérapeute. Elle rend de ce fait possible une «formalisation » des éléments thérapeutiques dans un manuel que le patient peut s'approprier lui-même. Selon ce point de vue, si chaque patient est « unique » dans un sens général, les manières de développer un trouble, par exemple dépressif ou anxieux, ne le sont pas. Il existe une grande régularité dans le fonctionnement psychique des troubles. Cette conception de l'homme induit que l'essentiel de la réalité psychique est, tout comme la réalité physique, « objectif » et partagé. Il est donc possible de décrire cette réalité dans des textes, y compris sur Internet. Le changement thérapeutique relève, selon l'approche comportementale, d'un processus d'apprentissage formalisable que le patient peut s'approprier plus ou moins indépendamment du thérapeute.

La conception selon laquelle un changement thérapeutique nécessite une relation thérapeutique est pleinement constitutive de la définition même de la psychothérapie traditionnelle. Par conséquent, une grande partie des thérapeutes et des chercheurs en psychologie clinique ne souhaitent pas la mettre en cause. Cette remarque vaut aussi pour la plus part des thérapeutes comportementaux et cognitivistes qui exercent aujourd'hui le plus souvent dans un cadre pratique et institutionnel très semblable à celui de la thérapie psychanalytique. La TCC (Thérapie Comportementale et Cognitive) moderne se déroule en effet généralement au moyen d'un suivi en face à face et sur la base de rendez-vous hebdomadaires dans un cabinet. En d'autres termes, si les racines théoriques du traitement psychologique par Internet résident dans la tradition comportementale, ce nouveau type de traitement pose aujourd'hui un défi très important à l'ensemble du champ des psychothérapies traditionnelles, et une grande partie des TCC inclus.

Le premier défi est théorique : si la thérapie s'appuyant sur Internet est aussi efficace que la thérapie dispensée d'une manière traditionnelle - nous avons vu que plusieurs études cliniques semblent effectivement le montrer -, un tel constat ébranle ce qui traditionnellement, et surtout dans la tradition psychanalytique, a été considéré comme le fondement de la psychothérapie : la relation unique entre un patient et son thérapeute. Les études cliniques conduites sur les traitements par Internet suggèrent que l'effet des thérapies s'explique plus par les techniques thérapeutiques utilisées que par la manière dont celles-ci sont dispensées.

Le défi théorique peut ainsi se résumer en deux questions. Qu'est-ce qu'une psychothérapie ? De quoi dépend son efficacité et comment mesurer cet effet ? Il serait exagéré d'affirmer que le traitement par Internet répond définitivement à ces défis théoriques fondamentaux pour la discipline. D'un point de vue psychanalytique, on 
serait naturellement critique envers la conception qui sous-tend les thérapies par Internet ainsi qu'envers la manière dont on prétend pouvoir les évaluer. La principale contribution théorique de ces nouvelles thérapies est peut-être, finalement, de faire apparaitre plus clairement ces questions fondamentales, qui ont été brouillées tant que les processus et les effets des thérapies comportementales et cognitives ont été étudiés sous la même « forme » que ceux des thérapies psychanalytiques.

Le deuxième défi est économique et institutionnel : si la psychothérapie a vocation à être de plus en plus dispensée sur Internet et basée sur des programmes de self-help guidés, les conséquences pour le secteur professionnel concerné seront importantes. Le cadre théorique traditionnel de la psychothérapie fonde de fait la psychothérapie en tant que secteur économique : aujourd'hui, une activité économique aux horaires de consultations tarifés, dans des cabinets. Dans certains pays, comme la Suède, le traitement psychologique par Internet a été introduit dans le système de santé mentale public. Dans les hôpitaux concernés, chaque thérapeute peut suivre environ quatre fois plus de patients à travers les programmes de soin par Internet qu'à travers la thérapie transmise en face à face (Bergström et al., 2010). Cela signifie que près de quatre fois plus de patients ont aujourd'hui accès à un traitement psychologique qu'avant la mise en œuvre de ces programmes.

En conclusion, cet article a présenté un nouveau domaine de la psychothérapie, qui a connu un développement important dans les cinq dernières années. Les effets définitifs que ce développement aura sur la psychothérapie dans son ensemble restent à observer. Une chose semble cependant acquise: on ne pourra plus penser la psychothérapie, ni organiser sa mise en œuvre, de la même manière qu'avant l'avènement des nouvelles thérapies par Internet.

\section{BIBLIOGRAPHIE}

ANDERSSON Gerhard, BERGSTRÖM Jan, BUHRMAN Monica, CARLBRING Per, HOLLÄNDARE Fredrik, KALDO Viktor, NILSSON-IHRFELT Elizabeth, PAXLING Björn, STRÖM Lars \& WAARA Johan (2008). “Development of a New Approach to Guided Self-Help via the Internet. The Swedish Experience”, Journal of Technology and Human Services, 26, pp. 161-181.

ANDERSSON Gerhard (2009). "Using the Internet to Provide Cognitive Behaviour Therapy”, Behaviour Research and Therapy, 47 (3), pp. 175-180.

ANDERSSON Gerhard \& CUIJPERS Pim (2009). “Internet-Based and Other Computerized Psychological Treatments for Adult Depression: A Meta-Analysis", Cognitive Behaviour Therapy, 38 (4), pp. 196-205.

ANDERSSON Erik, ENANDER Jesper, ANDRÉN Per, HEDMAN Erik, LJÓTSSON Brjánn, HURSTI Timo, BERGSTRÖM Jan, KALDO Viktor., LINDEFORS Nils, ANDERSSON Gerhard \& RÜCK Christian (2012). “Internet-Based Cognitive Behaviour Therapy for Obsessive-Compulsive Disorder: A Randomised Controlled Trial", Psychological Medicine, 42, pp. 2193-2203.

ALSTON Edwin (1962). "Bibliotherapy and Psychotherapy”, Library Trends, 11 (2), pp. 159-176. 
BAER Lee, GREIST John, \& MARKS ISaac (2007). "Computer-Aided Cognitive Behaviour Therapy", Psychotherapy and Psychosomatics, 76 (4), pp. 193-195.

BARLOW David (2002). Anxiety and Its Disorders: The Nature and Treatment of Anxiety and Panic, New York, Guilford Press.

BECK Aaron, RUSH John, SHAW Brian \& EMERY Gary (1979). Cognitive Therapy of Depression, New York, Guilford Press.

BENDELIN Nina, HESSER Hugo, DAHL Johan, CARLBRING Per, ZETTERQVIST NELSON Karin \& ANDERSSON Gerhard (2011). "Experiences of Guided Internet-Based Cognitive-Behavioural Treatment for Depression: A Qualitative Study", BMC Psychiatry, 11, pp. 107.

BERGSTRÖM Jan, ANDERSSON Gerhard, KARLSSON Andreas, ANDRÉEWITCH sergej, RÜCK Christian, CARLBRING Per \& LINDEFORS Nils (2009). "An Open Study of The Effectiveness of Internet Treatment for Panic Disorder Delivered in a Psychiatric Setting”, Nordic Journal of Psychiatry, 63 (1), pp. 44-50.

BERGSTRÖM Jan, ANDERSSON Gerhard, LJÓTSSON Brjánn, RÜCK Christian, ANDRÉEWITCH Sergej, KARLSSON Andreas, CARLBRING Per, ANDERSSON Erik \& LINDEFORS Nils (2010). “Internet- Versus GroupAdministered Cognitive Behaviour Therapy for Panic Disorder in a Psychiatric Setting: A Randomised Trial", BMC Psychiatry, 10, p. 54.

BRUSSET, Bernard (2012). "The Therapeutic Action of Psychoanalysis", The International Journal of Psychoanalysis, 93 (2), pp. 427-442.

CARR Anthony \& GHOSH Anindya (1983). "Response of Phobic Patients to Direct Computer Assessment", The British Journal of Psychiatry, 142, pp. 60-65.

CHRISTENSEN Helen, GRIFFITHS Kathy \& JORM Anthony (2004). “Delivering Interventions for Depression by Using the Internet: Randomised Controlled Trial”, British Medical Journal, 328 (7434), pp. 265-268.

CHRISTENSEN Helen, GRIFFITHS Kathy, MACKINNON Andrew \& BRITTLIFFE Kylie (2006). “Online Randomized Controlled Trial of Brief and Full Cognitive Behaviour Therapy for Depression”, Psychological Medicine, 36 (12), pp. 1737-1746.

CUIJPERS PIM, MARKS ISAac, VAN STRATEN Annemieke, CAVANAGH Kate, GEGA Lina \& ANDERSSON Gerhard (2009). “Computer-Aided Psychotherapy for Anxiety Disorders: A Meta-Analytic Review”, Cognitive Behaviour Therapy, 38 (2), pp. 66-82.

CUIJPERS PIM, DONKER TARa, VAN STRATEN Annemieke \& ANDERSSON Gerhard (2010). “is guided self-help as effective as face-to-face psychotherapy for depression and anxiety disorders ? A Meta-Analysis of Comparative Outcome Studies", Psychological Medicine, 40, pp. 1943-1957.

GHOSH Anindya, MARKS Isaac \& CARR Anthony (1984). "Controlled Study of Self-Exposure Treatment for Phobics: Preliminary Communication", Journal of the Royal Society of Medicine, 77 (6), pp. 483-487.

GOLDIAMOND Israel (1976). "Singling Out Self-Administered Behavior Therapies for Professional Overview. A Comment on Rosen”, American Psychologist, 31 (2), pp. 142-147.

GLASGOW Russell \& ROSEN Gerald (1978). "Behavioral Bibliotherapy: a Review of Self-Help Behavior Therapy Manuals”, Psychological Bulletin, 85 (1), pp. 1-23.

HEDMAN Erik, ANDERSSON Gerhard, LJÓTSSON Brjánn, ANDERSSON Erik, RÜCK christian, MÖRTBERG EWa \& LINDEFORS Nils (2011a). "Internet-Based Cognitive Behavior Therapy vs. Cognitive Behavioral 
Group Therapy for Social Anxiety Disorder: a Randomized Controlled Non-Inferiority Trial", PLoS One, 6 (3), e18001.

HEDMAN Erik, FURMARK TOMas, CARLBRING Per, LJÓTSSON Brjánn, RÜCK christian, LINDEFORS Nils, \& ANDERSSON Gerhard (2011b). "Five-Year Follow-up of Internet-Based Cognitive Behaviour Therapy for Social Anxiety Disorder”, Journal of Medical Internet Research, 13 (2), e39.

HOHL Eléonore, BERGER Thomas, BERGSTRÖM Jan, ANDERSSON Gerhard \& CASPAR Franz (2010).

"Caractéristiques et évidences empiriques des traitements psychologiques basés sur Internet", Journal de thérapie comportementale et cognitive, 20 (1), pp. 2-10.

KENWRIGHT Mark, LINESS sheena \& MARKS Isaac (2001). "Reducing Demands on Clinicians by Offering Computer-Aided Self-Help for Phobia/Panic. Feasibility Study", The British Journal of Psychiatry, 179 (5), pp. 456-459.

KLEIN Marjorie, GREIST John \& VAN CURA Lawrence (1975). “Computers and Psychiatry. Promises to Keep”, Archives of General Psychiatry, 32 (7), pp. 837-843.

LEWINSOHN Peter, ANTONUCCIO David, STEINMETZ BRECKENRIDGE Julia \& TERI Lin (1984). The Coping with Depression Course. A Psychoeducational Intervention for Unipolar Depression, Eugene, Castalia Publishing.

LY Hoa, CARLBRING Per \& ANDERSSON Gerhard (2012). "Behavioural Activation-Based Guided Self-Help Treatment Administered through a Smartphone Application: Study Protocol for a Randomized Controlled Trial", Trials, 13 (1), pp. 62-68.

PALMILI LUCa \& MARKS Isaac (2009). "Post-RCT Implementation of FearFighter in Primary Care Trusts across England", Paper presented at the 1st International E-Mental Health Summit, Amsterdam. PERINI Sarah, TITOV Nickolai \& ANDREWS Gavin (2009). "Clinician-Assisted Internet-Based Treatment is Effective for Depression: Randomized Controlled Trial", Australian and New Zealand Journal of Psychiatry, 43 (6), pp. 571-578.

PROUDFOOT Judith, RYDEN clash, EVERITT Brian, SHAPIRO David, GOLDBERG David, MANN Anthony, TYLEE Andre, MARKS Isaac, \& GRAY Jeffrey (2004). "Clinical Efficacy of Computerised Cognitive Behavioural Therapy for Anxiety and Depression in Primary Care: Randomised Controlled Trial", The British Journal of Psychiatry, 185, pp. 46-54.

MANHAL-BAUGUS Monique (2001). "E-therapy: Practical, Ethical and Legal Issues”, Cyberpsychology \& Behaviour, 4 (5), pp. 551-563.

MARTELL christopher, ADDIS Michael \& JACOBSON Neil (2001). Depression in Context. Strategies for Guided Action, New York, W. W. Norton.

MURAN christopher \& BARBER, Jacques (2010). The Therapeutic Alliance, New York, Guilford Press. NORTON Peter \& PRICE Esther (2007). “A Meta-Analytic Review of Adult Cognitive Behavioral Treatment Outcome across the Anxiety Disorders", Journal of Nervous and Mental Disease, 195 (6), pp. 521-531.

PALMQVIST Björn, CARLBRING Per, \& ANDERSSON Gerhard (2007). "Internet-Delivered Treatments with or without Therapist Input: Does The Therapist Factor Have Implications for Efficacy and Cost?", Expert Review of Pharmacoeconomics \& Outcomes Research, 7 (3), pp. 291-297.

RASSAU Anoushka \& ARCO Lucius (2003). "Effects of Chat-Based On-Line Cognitive Behavior Therapy on Study Related Behavior and Anxiety", Behavioural and Cognitive Psychotherapy, 31 (3), pp. 377-381. 
ROSEN Gerald, GLASGOW Russell \& BARRERA Manuel (1976). “A Controlled Study to Assess The Clinical Efficacy of Totally Self-Administered Systematic Desensitization”, Journal of Consulting and Clinical Psychology, 44 (2), pp. 208-217.

RUSKIN Paul, SILVER-AYLAIAN Michele, KLING Mitchel, REED susan, BRADHAM Douglas, HEBEL Richard, BARRETT David, KNOWLES Frederick \& HAUSER Peter (2004). “Treatment Outcomes in Depression : Comparison of Remote Treatment Through Telepsychiatry to In-Person Treatment", American Journal of Psychiatry, 161 (8), pp. 1471-1476.

STEWART Rebecca \& CHAMBLESS Dianne (2009). "Cognitive-Behavioral Therapy for Adult Anxiety Disorders in Clinical Practice: A Meta-Analysis of Effectiveness Studies", Journal of Consulting and Clinical Psychology, 77 (4), pp. 595-606.

TITOV Nickolai, ANDREWS Gavin, CHOI Isabella, SCHWENCKE Genevieve \& MAHONEY Alison (2008). "Shyness 3: An RCT Of Guided Versus Unguided Internet Based CBT For Social Phobia", Australian and New Zealand Journal of Psychiatry, 42 (12), pp. 1030-1040.

TITOV Nickolai, ANDREWS Gavin, JOHNSTON Luke, ROBINSON Emma \& SPENCE Jay (2010). “Transdiagnostic Internet Treatment for Anxiety Disorders: A Randomized Controlled Trial", Behaviour Research and Therapy, 48 (9), pp. 890-899.

WEIZENBAUM Joseph (1966). "ELIZA - A Computer Program For the Study of Natural Language Communication Between Man And Machine”, Communications of the ACM, 9 (1), pp. 36-45.

WESTEN Drew, NOVOTNY Catherine \& THOMPSON-BRENNER Heather (2004). “The Empirical Status of Empirically Supported Psychotherapies: Assumptions, Findings and Reporting in Controlled Clinical Trials", Psychological Bulletin, 130 (4), pp. 631-663.

WIMS Edward, TITOV Nickolai, ANDREWS Gavin \& CHOI Isabella (2010). "Clinician-assisted Internetbased treatment is effective for panic: A randomized controlled trial", Australian and New Zealand Journal of Psychiatry, 44 (7), pp. 599-607.

\section{NOTES}

1. Les deux termes sont ici utilisés en tant que synonymes.

2. Pour un examen plus détaillé, en français, des caractéristiques pratiques et des études d'efficacité des traitements psychologiques par Internet pour différents troubles psychologiques, nous renvoyons à l'article de Hohl et al. (2010).

\section{RÉSUMÉS}

Cet article propose une synthèse du développement scientifique du traitement psychologique par Internet, en explicitant et discutant ses traits caractéristiques. L'auteur y compare ce type de soin avec la psychothérapie traditionnelle et dessine les enjeux que revêtent les traitements sur Internet dans le champ de la psychothérapie; avant tout au niveau théorique mais aussi au niveau pratique. Plus précisément, il met en évidence les nombreux facteurs indiquant que les 
thérapies utilisant Internet constituent, cent ans après la naissance de la discipline, un défi profond et durable pour la psychothérapie.

This article provides an overview and discussion of the scientific developments in Internet-based psychological treatment. The author compares these therapeutic forms with those of traditional psychotherapy and delves into the issues this raises in the field of psychotherapy, primarily at a theoretical level, but also in practical terms. Specifically, the paper highlights the many factors indicating that a century after the discipline's birth, online therapies pose a fundamental challenge for psychotherapy.

\section{INDEX}

Keywords : therapy, Internet-based therapy, cognitive behavioral therapy, self-help, internetbased psychological treatment

Mots-clés : thérapie, thérapie par Internet, thérapies cognitivo-comportementales, self-help, traitement psychologique par Internet

\section{AUTEUR}

\section{JAN BERGSTRÖM}

Université de Stockholm, Département de psychologie 